\title{
Computerized tomography-guided percutaneous extralemniscal myelotomy
}

\section{Yücel Kanpolat, M.D., Ali Savas, M.D., Sükrü Çaglar, and Serdar Akyar, M.D.}

Department of Neurosurgery and Radiology, University of Ankara, School of Medicine, Ankara, Turkey

Extralemniscal myelotomy (ELM) is a procedure performed at the cervicomedullary junction of the spinal cord in which the central cord is lesioned to treat intractable pain. The neurophysiological mechanism of pain relief after ELM remains unclear. The authors present a series of 14 patients with intractable cancer pain who were managed by CT-guided, percutaneous ELM. In six of the cases (42.8\%), total pain relief was achieved; partial satisfactory pain relief was attained in four cases (28.5\%), and no pain control was achieved in four cases (28.5\%). No complications due to ELM were observed. The authors believe that ELM is a safe and effective procedure in the management of intractable cancer pain for selected cases; computerized tomography guidance is an essential part of the procedure to achieve morphological localization of the target in the cervicomedullary junction. More research is needed to understand the neurophysiological mechanism of pain relief after ELM and to standardize the lesion volume.

\section{Key Words * extralemniscal myelotomy * cervicomedullary junction * cancer pain * stereotaxy}

Extralemniscal myelotomy (ELM) is a neurosurgical operation performed to treat pain, and consists of the creation of centromedullary, stereotactic, electrolytic lesions near the cervicomedullary junction.[5,13,16] This operation was first performed by Hitchcock[5] in 1968 to destroy the upper cervical commissural fibers and attain analgesia in a patient suffering from pain in his neck and both arms caused by an esophageal adenocarcinoma. Most patients experience excellent pain relief and no clear-cut sensory loss on neurological examination after ELM. The procedure was also found to be effective for pain located in the lower portion of the trunk and lower extremities, as well as for visceral pain, central pain, and pain in the midline of the body. Since then, the indications for ELM have been broadened, although the neurophysiological mechanisms of pain relief remain unclear. Schvarcz[13] stated that the pain relief mechanism may be related to interruption of an extralemniscal, multisynaptic, nonspecific ascending system. Therefore, he named the procedure "extralemniscal myelotomy." Papo and Luongo[11] modified the operation by performing a laminectomy at the C1-3 level combined with deep electrocoagulation and sharp splitting of the posterior columns.

Conventionally, all percutaneous ELM procedures have been performed using x-ray visualization and myelography.[1,5,13] In spite of the use of contrast material, $x$-ray films have severe technical limitations related to visualization and are often unable to localize the electrode systems in the central cord region.[12] Topographical localization of the radiofrequency needle-electrode system in the 
cervicomedullary region is more easily visualized using computerized tomography (CT) guidance.[7,10] We report the technique and results of CT-guided percutaneous ELM performed in a series of 14 patients.

\section{CLINICAL MATERIAL AND METHODS}

Between 1986 and 1996, 14 patients suffering from intractable, subdiaphragmatic cancer pain were admitted to the Department of Neurosurgery at the University of Ankara Medical School. Four of the 14 patients had rectal carcinoma, four had pancreatic, two had gastric, two had colonic, one had hepatic, and one had renal cell carcinoma. In all cases, the pain was located in the lower trunk bilaterally and/or at the midline. Computerized tomography-guided ELM was indicated in all 14 patients.

Each patient undergoing ELM fasted for 5 hours before surgery. Seven milliliters of contrast material (Iatrolan or Iohexol) was administered into the subarachnoid space via lumbar puncture 20 minutes before the operation. The patient was placed prone on the CT table. The head was flexed and fixed. General anesthesia was not used; all patients were awake and cooperative throughout the procedure to facilitate observation of neurological functions.

In the first group of seven patients, Rosomoff's cordotomy electrodes with diameters of $0.45 \mathrm{~mm}$ and 1-mm open tips were used (model RCK-2A; Radionics, Inc., Burlington, MA) under CT guidance. In the second group of seven patients, a special needle electrode system was used for CT-guided procedures (KCTE Kit; Radionics, Inc.). To use this system, No. 20-22 gauge thin-walled needles with plastic hubs were designed to avoid imaging artifacts. We used an electrode with a $0.25-\mathrm{mm}$ diameter and 1-mm open tip in six cases and an electrode with a diameter of $0.25 \mathrm{~mm}$ and 2-mm open tip in one case.

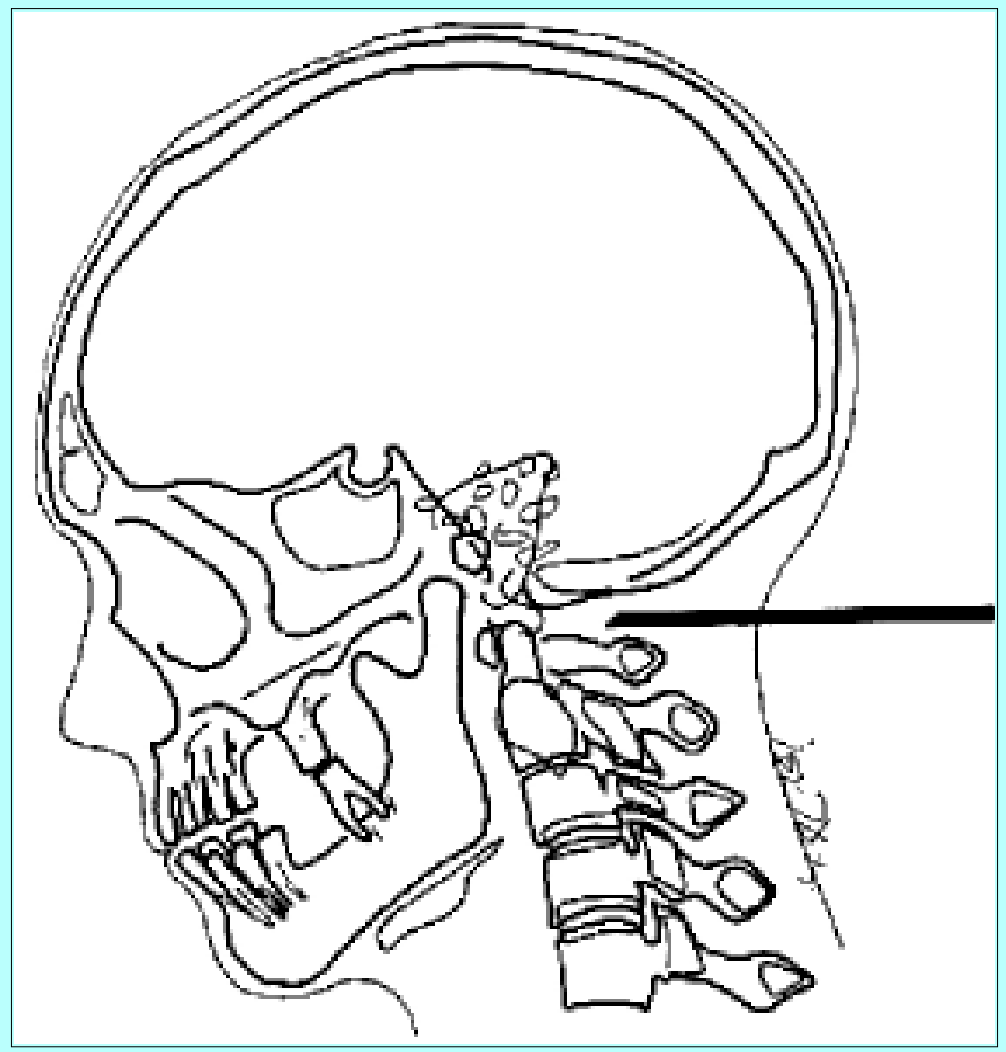

Fig. 1. Schematic drawing, lateral view, of the needle positioned in the cervicomedullary junction.

The needle was inserted at the C1-occiput level through the midline (Fig. 1). The target in ELM is the 
center of the spinal cord at the C1-occiput level (Fig. 2). The distance between the dura and skin was measured with CT slices, and it was found to range between $40.5 \mathrm{~mm}$ and $56.5 \mathrm{~mm}$ in 14 cases (mean 49 $\mathrm{mm}$ ). Measurements of the diameter of the spinal cord were made, and the active electrode was adjusted accordingly. Topographic localization of the needle was defined with the aid of axial CT scans.

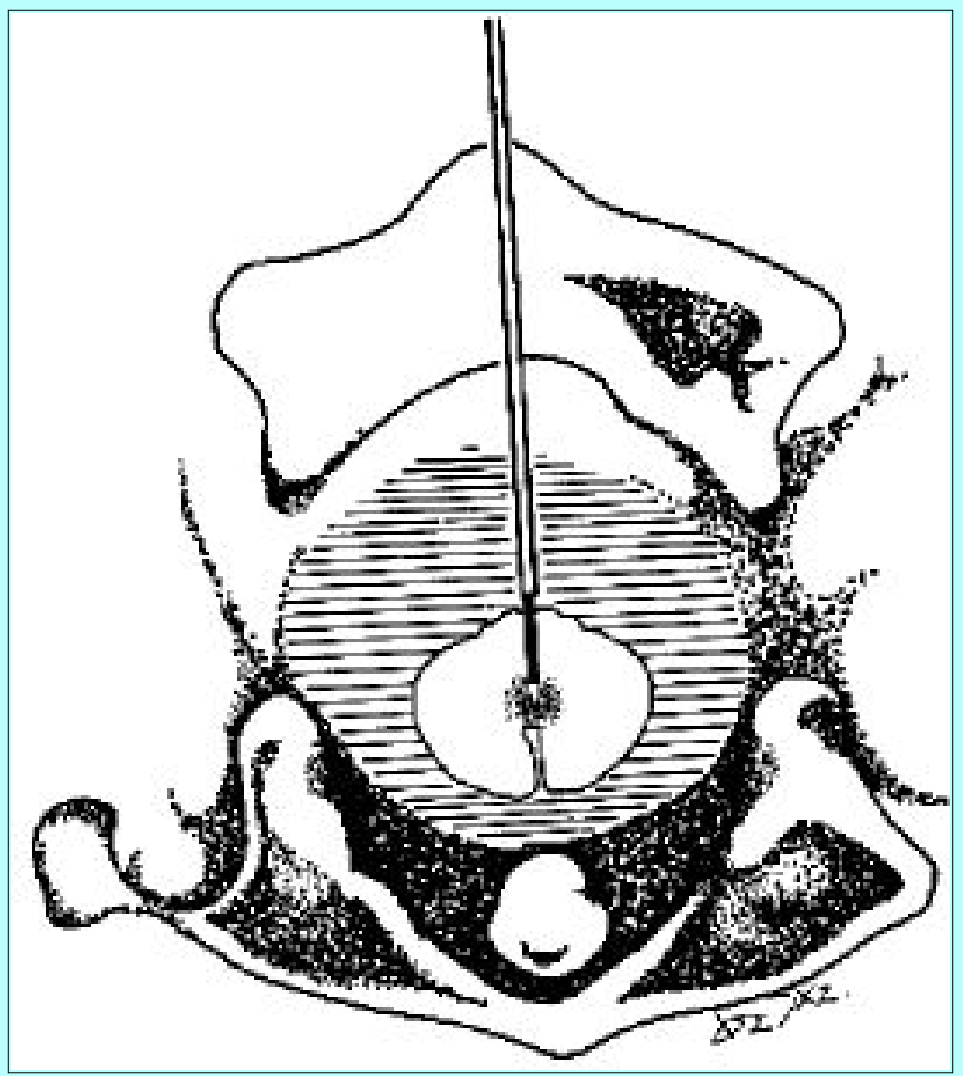

Fig. 2. Schematic drawing, axial view, of the needle electrode system located in the central cord at the cervicomedullary junction.

The needle was manipulated toward the center of the spinal cord with the help of a lateral scanogram and axial CT slices. Finally, the needle was approximated just behind the cord. The adjusted, active electrode tip was inserted into the cord via the needle (Fig. 3). Impedance measurements were taken, and stimulation was given for physiological control of the position of the active electrode. Impedance measurements were less than $400 \mathrm{Ohms}$ in the cerebrospinal fluid and greater than $1000 \mathrm{Ohms}$ inside the spinal cord. In most cases, paresthesia of the distal lower limb was observed with stimulation $(50-75 \mathrm{~Hz}$, $0.2-0.5 \mathrm{~V}$ ). The final controlled lesion was made at 70 to $80^{\circ} \mathrm{C}$ for 60 seconds; two lesions were usually made.

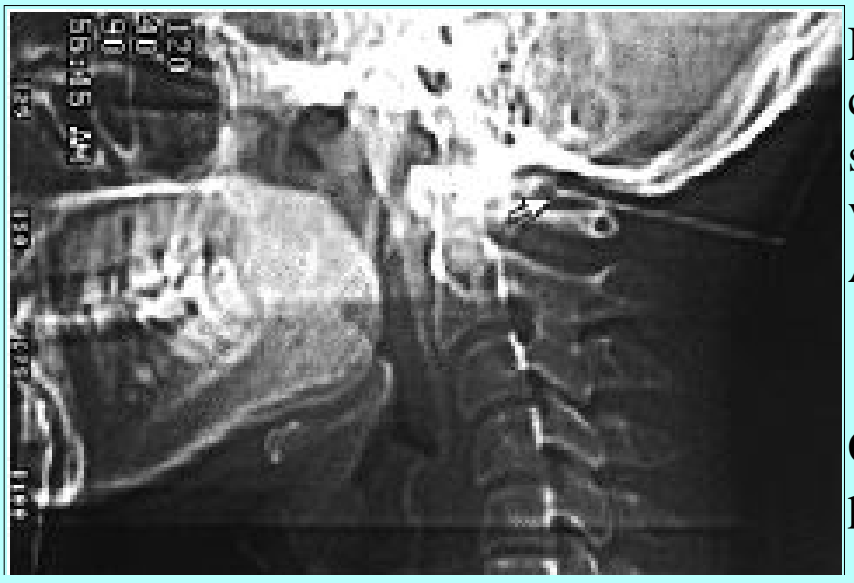

Fig. 3. Computerized tomography scans in the cervicomedullary junction during ELM. Upper: Lateral scanogram and position of the needle. Center: Axial view showing the final position of the needle. Lower: Axial view showing the final position of the electrode.

\section{RESULTS}

Computerized tomography-guided percutaneous ELM was performed 16 times in 14 patients. In six of the cases, total 


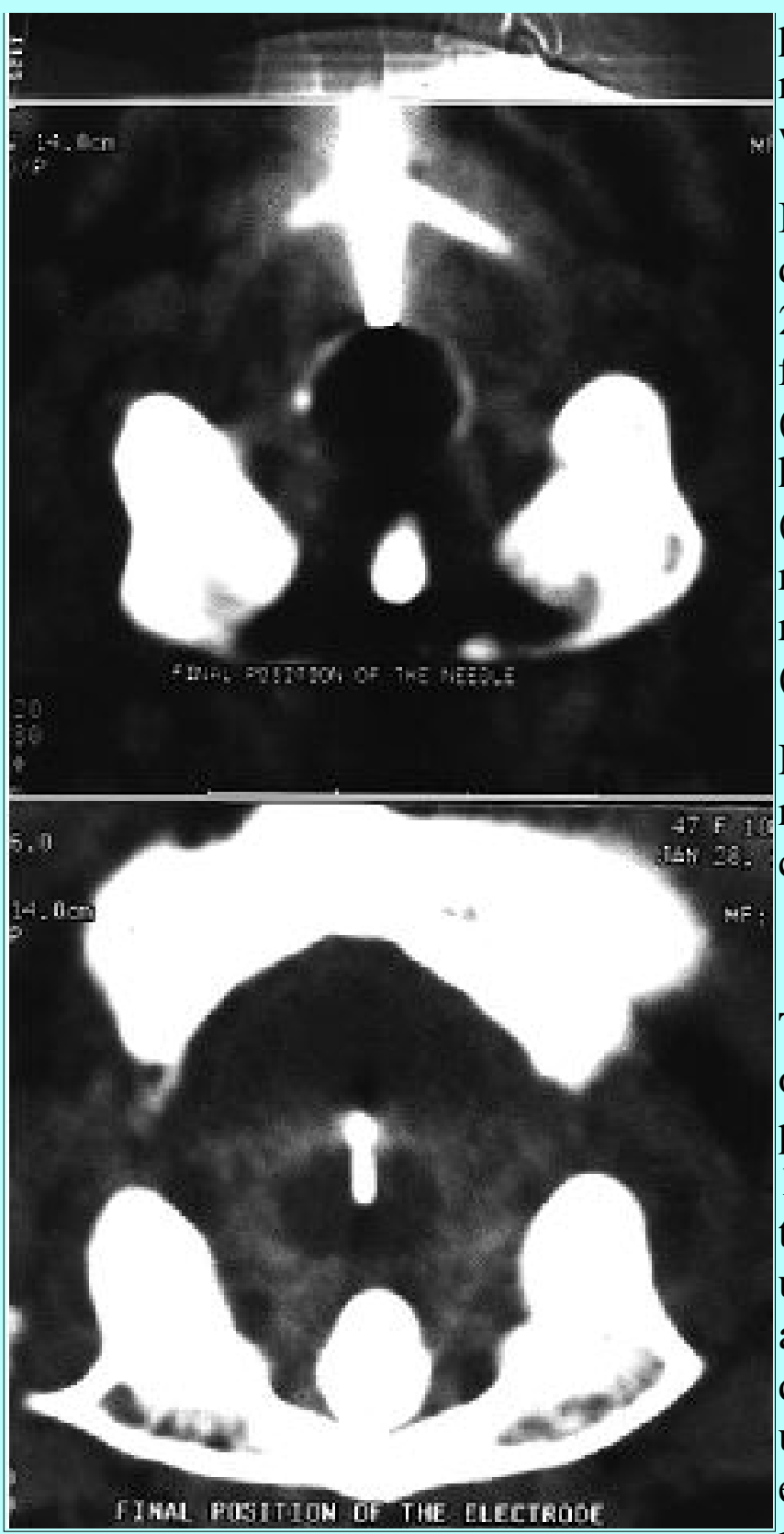

pain relief was achieved (42.8\%); partial, satisfactory pain relief was attained in four cases $(28.5 \%)$, and no pain control was achieved in four cases $(28.5 \%)$.

In eight patients in whom the larger electrodes $(0.45-\mathrm{mm}$ diameter with 1-mm open tip and $0.25-\mathrm{mm}$ diameter with 2-mm open tip) were used, total pain relief was achieved in five $(62.5 \%)$, partial satisfactory pain relief in two cases $(25 \%)$, and pain persisted in one case $(12.5 \%)$. On the other hand, in the six patients in whom the smaller electrodes (0.25-mm diameter with $1-\mathrm{mm}$ open tip) were used, total pain relief was achieved in one (16.6\%), partial satisfactory pain relief in two cases $(33.3 \%)$, and pain persisted in three cases $(50 \%)$.

Postoperative neurological examination of the patients revealed hypesthesia and hypalgesia in only one patient. No complications were observed.

\section{DISCUSSION}

The center of the spinal cord at the spinomedullary junction is one of the most important targets in pain surgery. The first procedure in this region was performed by Hitchcock[5] in 1968. His aim was to interrupt the decussating pain fibers at the cervicomedullar level to obtain pain relief in the neck and upper extremities. He used 0.5-mm diameter electrodes with a 1-mm open tip. Later procedures in the central spinal cord demonstrated that the lesions produced relief of pain in the upper body and extremities and in the lower body and lower extremities, including visceral pain, of pain located at the midline, and even of central pain.[5,6] These observations resulted in a change in the indications for central cord lesions. Schvarcz stated that "The procedure, however, was not aimed at severing segmental decussating fibers, but at interrupting selectively the extralemniscal system. That is an ascending nonspecific polysynaptic pathway."[13] He named the procedure "extralemniscal myelotomy." He used 0.5-mm diameter tungsten wires with a 2-mm bare end. The target for lesioning was described by Schvarcz as just in front of the dorsal funiculus where the distal lower limb threshold responses were obtained.[12,13] Eiras, et al.,[1] performed similar procedures using 0.5-mm diameter electrodes with a 2-mm bare end. Papo and Luongo[11] performed six to seven central cord lesions between the C1-3 segments using 0.4-mm diameter electrodes with a 2.5-mm open tip. Gildenberg and Hirshberg[2-4] performed limited myelotomy using an open technique at the T-10 level for similar purposes.

The target area typically described by the authors is the center of the spinal cord, just posterior to the center and in front of the posterior funiculus.[1,5,12,13] Lesions measuring 2.5 to 3-mm in diameter are the goal. It is almost certain that the effect on pain is not related to interruption of the commissural fibers. One explanation for the loss of pain in the lower body is that there exists an entirely separate pure pain 
pathway that ascends the spinal cord and is nonspecific, multisynaptic, and close to the midline, as stated by Hitchcock and Schvarcz. Shealy, et al.,[14] found bilateral activity elicited by $\mathrm{C}$ fibers in the central cord region. Additionally, extensive cross connections within the propiospinal system are densely grouped at the base of the dorsal funiculi in the same area.[7,13]

Ideal candidates for ELM are patients with intractable pain located in the midline or with bilateral involvement. This procedure is preferable particularly because of the absence of respiratory complications such as sleep-induced apnea.[6,7] Schvarcz[13] has recommended the procedure for central pain states as well. In the 114 cancer patients reported in the literature, $85 \%$ had good initial pain relief, whereas long-term pain relief was achieved in less than $50 \%$ of cases, as reported by Sindou, et al.[15] The overall results of various studies are presented in Table 1.

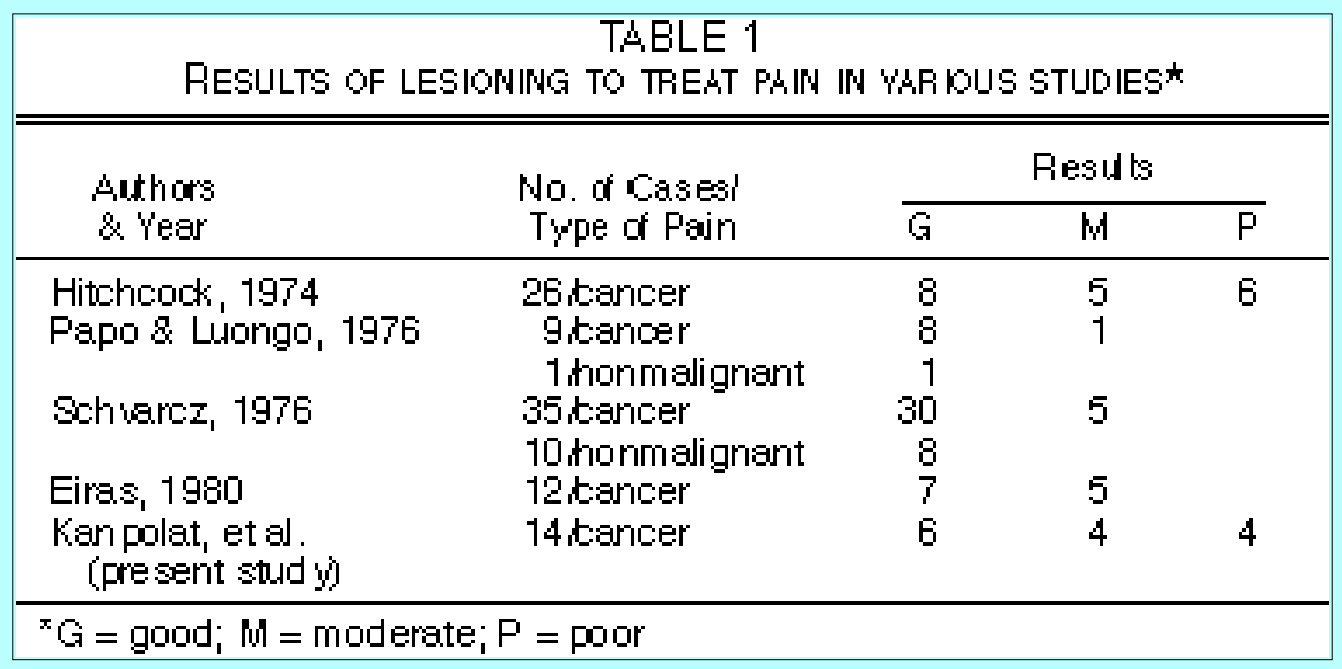

Extralemniscal myelotomy has traditionally been performed with the aid of x-ray visualization. In spite of the use of contrast materials, it is almost impossible to identify the target area. Because the diameters of the spinal cord change from patient to patient, it is inappropriate to use standard-length electrode tips. Our clinical and experimental studies have shown that the spinal cord diameters at the level of the C1-occipit range from 7 to $12.8 \mathrm{~mm}$ sagitally, and 9.3 to $14 \mathrm{~mm}$ transversely;[9] thus, insertion of the electrode tip should be adjusted according to the spinal cord diameters measured by CT visualization. Another advantage of CT guidance is that it allows visualization of the active electrode location in the spinal cord.[7,8] The tip of the active electrode may be redirected if needed during the procedure, according to the images.

The first group of operations were performed using electrodes with a diameter of $0.45-\mathrm{mm}$ and $1-\mathrm{mm}$ open tip. Later, we used smaller thermocoupled electrodes with a $0.25-\mathrm{mm}$ diameter and $1-\mathrm{mm}$ open tip. However, the results of procedures performed with the smaller electrodes were poor; we recently changed our electrode dimensions to 0.4-mm diameter and 2-mm open tip.

Extralemniscal myelotomy consists of three basic steps. The first step is to visualize the anatomy and morphology of the target by CT scanning and then to make our approach using the needle electrode system. The second step is to gain neurophysiological control of the target area by impedance measurements and electrical stimulation. The final step is to create controlled, defined, radiofrequency lesions while monitoring neurological functions.

Based on the results of our limited series, ELM is an effective and safe procedure. Poor results were obtained mostly in procedures in which the smaller electrodes were used. We believe that ELM is a 
neglected but valuable and effective treatment of well-classified cancer pain located in the midline and/or bilaterally. The essentials of the procedure are CT-guided morphological targeting, impedance measurements, and stimulation for neurophysiological control, and controlled defined lesioning. In our opinion, the use of ELM in the treatment of intractable pain will increase with a better understanding of the pertinent anatomical structures and standardization of the lesion volume.

\section{Acknowledgments}

We express our appreciation to Ms. Helen Stevens for editing this article and to Dr. Ali Oguz Tasçioglu for his creative drawings.

\section{References}

1. Eiras J, Garcia J, Gomez J, et al: First results with extralemniscal myelotomy. Acta Neurochir Suppl 30:377-381, 1980

2. Gildenberg PL: Myelotomy and percutaneous cervical cordotomy for the treatment of cancer pain. Appl Neurophysiol 47:208-215, 1984

3. Gildenberg PL, Hirshberg RM: Limited myelotomy for the treatment of intractable cancer pain. $\mathbf{J}$ Neurol Neurosurg Psychiatry 47:94-96, 1984

4. Hirschberg RM, Al-Chaer ED, Lawand NB, et al: Is there a pathway in the dorsal funniculus that signals visceral pain? in Brock M, Fuhrmann H, Hoell T, et al (eds): The Abstract Book of the 10th European Congress of Neurosurgery. Berlin: Hellmich, 1995, p 176

5. Hitchcock E: Stereotactic cervical myelotomy. J Neurol Neurosurg Psychiatry 33:224-230, 1970

6. Hitchcock E: Stereotactic myelotomy. Proc R Soc Med 67:771-772, 1974

7. Kanpolat Y, Akyar S, Çaglar S: Diametral measurements of the upper spinal cord for stereotactic pain procedures: experimental and clinical study. Surg Neurol 43:478-483, 1995

8. Kanpolat Y, Atalag M, Deda H, et al: CT guided extralemniscal myelotomy. Acta Neurochir 91:151-152, 1988

9. Kanpolat Y, Çaglar S, Akyar S, et al: CT-guided pain procedures for intractable pain in malignancy. Acta Neurochir Suppl 64:88-91, 1995

10. Kanpolat Y, Cosman E: Special RF electrode system for CT-guided pain procedures. Neurosurgery 38:600-603, 1996

11. Papo I, Luongo A: High cervical commissural myelotomy in the treatment of pain. J Neurol Neurosurg Psychiatry 39:705-710, 1976

12. Schvarcz JR: Functional exploration of the spinomedullary junction. Acta Neurochir Suppl 24:179-185, 1977

13. Schvarcz JR: Stereotactic extralemniscal myelotomy. J Neurol Neurosurg Psychiatry 39:53-57, 1976 
14. Shealy CN, Tyner CF, Taslitz N: Physiological evidence of bilateral spinal projections of pain fibers in cats and monkeys. J Neurosurg 24:708-713, 1966

15. Sindou M, Jeanmonod D, Mertens P: Ablative neurosurgical procedures for the treatment of chronic pain. Neurophysiol Clin 20:399-423, 1990

16. Sweet WH, Poletti CE: Operations in the brain stem and spinal canal, with an appendix on open cordotomy, in Wall PD, Melzack R (eds): Textbook of Pain, ed 2. Edinburgh: Churchill Livingstone, 1989, pp 819-820

Manuscript received November 20, 1996.

Accepted in final form December 18, 1996.

Address reprint requests to: Yücel Kanpolat, M.D., Inkilap Sk. 24/2, Kizilay 06650 Ankara, Turkey 